\title{
Magnetic and microwave properties of polycrystalline gadolinium iron garnet
}

\begin{abstract}
The microwave loss in nanosized GdIG particles synthesized using mechanical alloying technique was investigated. There were very few of research on the microwave properties of nanosized particle GdIG and there is no attempt investigating on the material at C-band frequency range and its correlation with the microstructure. Gadolinium (III) iron oxide and iron (III) oxide, $\alpha$-Fe2O3 were used as the starting materials. The mixed powder was then milled in a high-energy ball mixer/mill SPEX8000D for 3 hours. The samples were sintered at temperature $1200^{\circ} \mathrm{C}$ for 10 hours in an ambient air environment. The phase formation of the sintered samples was analyzed using a Philips X'Pert Diffractometer with $\mathrm{Cu}-\mathrm{K} \alpha$ radiation. Complex permeability constitutes of real permeability and magnetic loss factor were measured using an Agilent HP4291A Impedance Material Analyzer in the frequency range from $10 \mathrm{MHz}$ to $1 \mathrm{GHz}$. A PNA-N5227 Vector Network Analyzer (VNA) was used to obtain the information on ferromagnetic linewidth broadening, $\Delta \mathrm{H}$ that represents the microwave loss in the samples in in frequency range of 4 to $8 \mathrm{GHz}$ (C-band). The $\Delta \mathrm{H}$ value was calculated from the transmission (S21) data acquired from VNA. The single phase GdIG showed low initial permeability and low magnetic loss when applied with low-frequency range energy. From these data, it is validated that GdIG is a suitable material for microwave devices for the high-frequency range.
\end{abstract}

Keyword: Gadolinium iron garnet; Permeability; Magnetic loss; Ferromagnetic linewidth 